\title{
Managing Complex Work Systems via Crowdworking Platforms: The Case of Hamburger Hochbahn and Phantominds
}

\author{
Volkmar Mrass \\ University of Kassel \\ volkmar.mrass@uni-kassel.de
}

\author{
Christoph Peters \\ University of St.Gallen \\ and University of Kassel \\ christoph.peters@unisg.ch
}

\author{
Jan Marco Leimeister \\ University of St.Gallen \\ and University of Kassel \\ janmarco.leimeister@unisg.ch
}

\begin{abstract}
In the last decade, crowdsourcing has emerged as a new form of work organization. Crowdworking platforms as intermediaries between crowdsourcing companies and crowd workers have gained importance in this process. Currently, many of these platforms manage rather simple work systems. Using the case of the German Hamburger Hochbahn $A G$ and the innovation platform Phantominds, this paper investigates measures necessary for crowdworking platforms to be able to manage also more complex work systems. To derive such measures, we analyze the work system of Hamburger Hochbahn and Phantominds, explore the interplay between the crowd and the platform provider and subsequently provide recommendations for companies that would like to use crowdworking platforms for the processing of work and for platform operators. With this paper, we extend current knowledge in the realms of IS, organizational theory, and platform ecosystems.
\end{abstract}

\section{Introduction}

Although crowdsourcing is not a new phenomenon [1], using what is perceived as the "wisdom of crowds" [32] has received a boost by the new possibilites provided via the Internet. The wisdom of crowds is a very specific phenomenon, based upon the aggregation of independent estimates about objectively measurable events [24], and therefore applies only to some types of crowdsourcing. Nevertheless, an increasing amount of companies and other institutions started to exploit crowdsourcing for their purposes in the last decade since Howe [14] coined this term. The platforms that act as intermediaries between crowdsourcing institutions and crowd workers have therefore gained in importance and can be used for a wide range of activities along their value chain [22]. If these platforms deal with the processing of paid work, it is reasonable to use the term 'crowdworking platforms' [21]. These platforms entail a new principle of work organization and a 'paradigmatic change': Work is not assigned to workers anymore; instead, they choose their work themselves [20]. URI: http://hdl.handle.net/10125/50405

ISBN: 978-0-9981331-1-9

(CC BY-NC-ND 4.0)
This is usually done after announcing work on such platforms via either an open call where all registered crowd workers are informed or a restricted call where a specific segment of the crowd is targeted.

Despite the increasing use of such crowdworking platforms, many of them are still focussed on work that is of rather simple nature (and as a consequence also on the management of rather simple work systems). The authors of this paper believe that there are at least three major reasons to explore how also complex work systems can be managed via such crowdworking platfoms: Firstly, the technological development will lead to increased "computerisation" of jobs (see e.g. [13], [8], [12]), meaning that more and more jobs will become susceptible to digitization. This makes it also more likely that rather simple work currently performed by humans on such platforms will be automated. Secondly, many crowdworking platforms are increasingly coming "under scrutiny" since several societal players (see e.g. [5], [9]) have started discussions about fair working conditions, "new Taylorism" (often associated with sweatshop work) or minimum wages. Processing more complex work would allow to pay higher wages and to meet potential future requirements which might be imposed by legislators. Thirdly, this business model simply offers more potential for the processing of work than it is currently the case. For example, an investigation of 32 crowdworking platforms [19] in Europe's largest economy, Germany, showed that the majority of them focusses on work such as collecting data from the point of sale (POS), designing t-shirts, microtasking, testing devices and software, writing short texts, or the like. Even though this is decent work that fits the business model of many platforms quite well, using the potential of crowdworking also for the management of more complex work would be a natural further development of the business model of such platforms and would make them even more attractive to companies.

All reasons mentioned above serve us as a motivation to investigate how complex work and the respective work systems can be managed via crowdworking platforms. This case study of ours is one step towards this goal, further steps with additional case studies about

\section{$\mathrm{H}$ C C S S}

Page 4112 
other projects are following. We believe that the complexity of work can serve as a good proxy for the complexity of a work system necessary to process that work and that both are highly correlated. Research regarding this topic is important for the future success of crowdworking platforms as a digital innovation of the last decade. We position our research in the realm of information systems (IS) and organizational theory, especially in the relatively new area of platform ecosystems. We aim at contributing to this realm and extending current knowledge by exploring measures for the successful processing of complex work systems via crowdworking platforms. To do so, we look at this issue on a more "macro-level", using the lense of work system theory (WST) [2] and investigating the interplay of participants, information and technologies to perform processes and activities with the aim to deliver products and services to the customers. With this paper, we aim at getting first insights about the management of complex work systems via crowdworking platforms by investigating an interesting project the crowdworking platform Phantominds conducted with Germany's second largest local public transportation provider Hamburger Hochbahn AG. In this paper, we pursue the following research question:

RQ: How can complex work systems successfully be managed via crowdworking platforms?

After the introduction, this paper proceeds as follows: First, we provide a foundational theoretical background. Second, we describe the research methodology and case selection for the conduction of our research. Third, we introduce Hamburger Hochbahn AG and Phantominds and their joint project. Fourth, we analyze the work system, communicate our findings and insights regarding the management of complex work systems via crowdworking platforms that we gained and derive recommendations. Finally, we close with a discussion and a conclusion and an outlook on our future research.

\section{Theoretical background}

For our research regarding the management of complex work systems via crowdworking platforms, there are different theoretical areas that deliver valuable contributions as background. On a general basis, especially the realm of organizational theory provides insights on the management of complex work systems: Scott and Davis [30] for example deliver a good overview of several approaches and theories that help to connect the area of complex systems in general with complex work systems managed via crowdworking platforms. Examples include Fayol's [11] top-down managerial approach to divide and coordinate complex work systems, Boulding's [7] classification of systems by their level of complexity, Beer's [4] classification of systems ranging from simple/deterministic over complex/propabilistic to exceedingly complex/probabilistic, Ashby's [3] notion that no complex system can only be understood by an analysis that attempts to decompose the system into its individual parts and Perrow's [23] view that with regard to complex, probabilistic systems, the whole is more than the sum of its parts. Particularly notable is also the view, communicated among others by Scott [29] and Stinchcombe [31], that one way to manage greater complexity is not to divide the work and distribute it among different workers but to tackle complexity with more highly qualified and flexible performers (professionals) and that as levels of complexity, uncertainty and interdependence increase, formerly independent professionals are likely to move their work into organizational structures.

Since the management of complex work systems via crowdworking platforms differs from the management in other settings (e.g. by the fact that the participants of the work system, especially the crowd workers, are often not known and the management of the system is done via an electronic platform), the area of platform ecosystems also offers relevant theoretical background for our research. Boudreau et al. [6] for example assess the main requirements for successful online team collaborations outside a company. They show how alternative organizational forms such as online collaborative platforms can coordinate the collective effort of creative workers to solve complex innovation problems. Drawing also on evidence in extant literature, the authors state that higher levels of emergent interdependence lead to higher quality solutions to complex, multi-faceted problems, even despite the loss of divergence in ideas that may occur as a result of interaction [6]. The authors also point out that the history of online collaborative platforms stresses the use of enabling technologies and processes that simply reduce coordination costs. Similarly, Tiwana et al. [33], p. 7) note that information technology (IT) has yielded formerly infeasible forms of organizational governance and that these new logics have at the same time reinforced the need for effective IT governance. They identify theoretical blind spots regarding IT governance research and note that only miniscule attention has been directed to larger-scale ecosystems of firms and systems so far ([33], p. 8). This is also the area where complex work systems managed via crowdworking platforms as our unit of analysis can be positioned and to which we aim at contributing with our ongoing research.

Prpić et al. [28] distinguish four types of crowdsourcing: crowd-voting where an organization requests choices between alternatives and then aggregates the votes, idea crowdsourcing where an organization invites opinions for small or big questions and then evaluates the proposed ideas, micro-task crowdsourcing where an organization breaks a problem into smaller jobs and then re-assembles the completed tasks, and solution crowdsourcing where an organization invites and tests contributions for specific problems and then adopts the best non-falsifiable solutions. In this paper, we focus on 
the second type (idea crowdsourcing). Regarding Crowd Science [26], the case described in our paper can be positioned in the crowdsourcing realm and there in the following way: 1 . The crowd exists outside the organization (Hamburger Hochbahn), Phantominds conducted an open (not a targeted) call and the size of the crowd has been around 5,000 members. 2. The crowd capability can be characterized by an IT structure that allows for highly collaborative work, mostly via the Web, and processes where the most promising ideas are filtered. Regarding the benefit Hamburger Hochbahn is seeking from Phantominds' IT-mediated crowd (crowd capital, see also [27]), our case can be characterized by more than 130 ideas that have been created. While our work focuses on crowdsourcing, other realms of Crowd Science [26] may also benefit from it: For example, our insights are also valuable for the realm of citizen science since a lot of the contributors from the crowd of Hamburger Hochbahn are at the same time citizens of Hamburg and the solutions developed have a direct impact on their lives. Crowdsourcing literature also distinguishes between crowd-IT that is found in the forms "episodic" and "collaborative" (e.g. [28], [25]). While in our case, both elements can be found, the latter is more prevalent. The episodic element is present since the contest for Hamburger Hochbahn on Phantomind's crowdworking platform is of limited duration. Nevertheless, the collaborative element is very strong since crowd workers collaborated when working on their solutions for Hamburger Hochbahn and this collaboration is according to our case study interviews a main success factor when it comes to the management of complex work systems.

In general, crowdsourcing platforms can be seen as intermediaries and the point where the controlling and management of the crowd and of all activities within the crowd take place [17]. If these crowdsourcing platforms focus on the processment of paid work, we use the term crowdworking platform [21].

In organizational environments, work is "the application of human, informational, physical, and other resources to produce products/services" ([2], p. 75). Sometimes, the terms work and task are used synonymously. In our research, we use the term "task" for a rather limited and narrow "to do" that is more likely (even though not necessarily) to be done by an individual without too much interaction with others. We use the term "work" as something that has a more holistic character/nature and is more likely to require collaboration, coordination and interaction with others. Complex work is for the authors of this paper - derived from the description of characteristics of simple work on a microtask platform by Kittur et al. [15] and reversing these - in general work that mostly requires coordination, a high level of cognitive effort, expertise and skills in the respective area, time and contextual information; it is usually heterogeneous, interdependent, rather non-repetitive and has multiple stakeholders. The World Bank uses in a study [16] skills and education or training required as a proxy to determine work complexity. It assigns low complexity to microwork where mostly no specialized skills or training are required and basic computer and Internet literacy (and the associated language) skills are usually sufficient. It attributes high complexity ([16], p. 13) to work from areas such as engineering, software development or human resources

A work system is "a system in which human participants and/or machines perform work (processes and activities) using information, technology, and other resources to produce specific products/services for specific internal and/or external customers" ([2], p. 75). In our case, participants of the work system are first of all the crowd workers (of Phantominds), but often also the customers (Hamburger Hochbahn) if they participate in the creation of the products and services, or the internal employees of the platform operator. Information refers to informational entities such as orders or invoices as well as to conversations and verbal commitments by the work system participants ([2], p.80). Technologies include both tools that are used by work system participants and automated agents (i.e. hardware and software configurations) since some work systems are totally automated (ibidem). Processes and activities occur in the work system to create products and services for its customers. Besides the elements of the work system itself described above, the Work System Framework ([2], p. 78) also includes environment, infrastructure, and strategies. As already mentioned in the introduction, the complexity of work serves us as a good proxy for the complexity of the work system necessary to process this work. With our research, we are looking at IT-reliant work systems; more specifically, at work systems that are managed via crowdworking platforms. We purposefully mostly use the term "manage" (in the sense of planning/steering/controlling) instead of "govern" since the landscape of crowdworking platforms is heterogenous and some platforms are more coordinating the supply and demand than really governing the whole work system (although the term govern would be accurate in many cases).

\section{Methodology and case selection}

To investigate our unit of analysis, the work systems, we employ a single-case study approach to shed more light on this issue that has not been in the main focus of IS research so far. According to Yin [34], the case study research method is in general especially useful when (1) the main research questions are "how" or "why" questions, (2) a researcher has little or no control over behavioral events and (3) the focus of study is a contemporary (not entirely historical) phenomenon. This is true regarding our investigation: With our research question, we strive to examine how complex work systems can be

Page 4114 
managed via crowdworking platforms; we have no influence on behavioral events since we do not interfere in the interactions between crowdsourcers, platforms and crowd workers and the focus of our study, crowdworking platforms, are a current phenomenon and not something from the past. In order to realize triangulation and thereby providing a stronger substantiation of constructs and hypotheses as suggested by Eisenhardt [10], we used the following multiple sources to collect data for this case study:

- In-depth interviews of about 1.5 hours each with the Managing Director of Phantominds, Dr. Mirko Bendig (on February $21^{\text {st }}, 2017$, and June $13^{\text {th }}$, 2017)

- Intense analysis of the information available on the Internet websites of Hamburger Hochbahn AG and Phantominds (February to June 2017)

- Analysis of other publicly available information (e.g. press) about the project (May to June 2017) (see for example: www.welt.de/regionales/hamburg/article150899281/Hochbahn-ist-auf-der-

Suche-nach-der-mobilen-Zukunft.html)

- Evaluation of additional material received from Phantominds after the interviews with the Managing Director Dr. Mirko Bendig (February and June 2017).

For the semi-structured interviews, we developed a guideline with questions addressing different areas of the work system (e.g. measures for the steering of the participants, the planing of the processes/activities, the design of the platform/technology, etc.) to get insights from different perspectives on the theme of managing complex work systems via crowdworking platforms such as Phantominds. The interviews were recorded and subsequently transcribed. A draft of the resulting paper has been delivered to the interviewee to offer the possibility to comment it or request adjustments if necessary. To be able to generate the desired insights, we chose a crowdworking platform that:

- Deals with the management of complex work (systems) as defined in section 2 ,

- Has already been existing for a while (i.e., at least three years) and therefore makes it more likely that it has gained enough expertise to answer our questions regarding the management of complex work systems adequately and that we are also able to study its development in the future,

- Has conducted projects that have been very successful (to be able to generate useful recommendations for projects of other companies and platforms).

We selected the crowdworking platform "Phantominds" since it manages such complex work systems. The platform is furthermore not restricted to the use in a specific company (which is the case with several proprietary platforms), but basically open to several kinds of companies that want to use its services. Despite the fact that the area of crowdworking companies is dynamic and many companies that existed years ago do not exist anymore (e.g. because they merged with other companies or went bankrupt), Phantominds has already been in the market for more than three years and proved to be able to "survive" in the long term. Moreover, the area of innovation services is in general an area that require the management of more complex work systems than it is for example the case with microtask platforms. Therefore, the examination of the seminal project managed via the crowdworking platform Phantominds with Hamburger Hochbahn proved to be valuable to answer our research question.

\section{The case of Hamburger Hochbahn and Phantominds}

\subsection{Company profiles}

Hamburger Hochbahn AG (short: "Hochbahn"), situated in Germany's second largest city Hamburg in the north of the country, is one of the oldest institutions of its kind in Europe. Founded in 1912, the company is currently the second largest local public transportation company in Germany with about 5,000 employees and 1.2 million passengers per day (see also: www.hochbahn.de). Hamburger Hochbahn AG operates 4 metro and 111 bus lines, serving 91 metro and 1,321 bus stations. The 232 rail vehicles and 803 buses transport more than 430 million passengers per year, completing around 2 billion kilometers (see also: www.hochbahn.de).

Phantominds UG is an innovation crowdworking platform based in Hamburg, Germany. Founded in 2014, the company has still retained its "start-up-mentality" while operating an increasing community of more than 10,000 crowd workers and serving customers from different industries. The main business goal of the company is to offer collaborative innovation services ("user generated innovations"). The company sees itself as an alternative to traditional business consultancies and agencies, providing access to its crowd community and direct dialogue possibilites to potential customers (see also: www.phantominds.com/ueber-uns).

\subsection{Initial situation}

The digitization of business and society has further proceeded in the last years. The competitiveness of whole countries, economies, branches and corporations depends on how successfully they are able to adapt to the present changes [18]. Encountering this trend, Hamburger Hochbahn AG started a comprehensive digitization process in 2016 (see also: www.hochbahn.de). It also has a company strategy "HOCHBAHN\#2030" in place that responds to the megatrend digitization and aims to explore new opportunities for the company. The 
major goal of this strategy is to successfully cater to the customer needs and further increase passenger numbers. Among the means to implement the strategy "Hochbahn\#2030" are creating new solutions for a new digital customer experience, exploring new business segments and fostering innovations for the customers (see also: www.hochbahn.de).

\subsection{The project}

Hamburger Hochbahn AG approached Phantominds with the aim to jointly develop new business models, offerings and services for its customers. The goal has been to make its public transportation services more attractive and to provide additional incentives to use its offerings. The ideas and innovations developed by the crowd should include the existing infrastructure of Hamburger Hochbahn with its 749 metro wagons, 803 busses and altogether 1.412 stations. One main aspect in this context has been the fact that Hamburger Hochbahn's customers (the "consumers") spend a lot of time driving or waiting and that they should be provided with offerings and services to use that time. An idea Hamburger Hochbahn provided in advance (to kick off the contest) has been to let the crowd workers think what services of other companies they could include, following the example of their already existing cooperation with the parcel delivery company "Hermes" (see also: http://bit.ly/1i8LNxB). The four concrete questions that aimed at providing a guideline for the Phantominds crowd have been:
- How can Hamburger Hochbahn support passengers to better use their travel and waiting time?

- How can one provide interesting and useful information around the transportation network?

- Which activity and entertainment possibilities are thinkable?

- Which shopping options could be provided for the customers ("last mile shopping")?

The customer emphasized that ideas developed by Phantominds's crowd should be feasible on the basis of the already existing infrastructure of Hamburger Hochbahn (i.e., without the need for constructional changes).

\section{Findings and insights}

In this section, we will depict the core findings and insights gained from this case regarding the management of complex work systems via crowdworking platforms. Firstly, we will analyze the work system of Hamburger Hochbahn and Phantominds as a basis for both the better understanding how Phantominds manages it and the subsequent derivation of our recommendations (section 5.1). Secondly, we will investigate the general process used by Phantominds to generate ideas for products and services (section 5.2). Thirdly, we will shortly introduce the Lean Integrated Innovation (LIC) Method of Phantominds (section 5.3) before we describe the approach used by Phantominds during the project with Hamburger Hochbahn (section 5.4). Finally, we derive measures and recommendations for the management of complex work

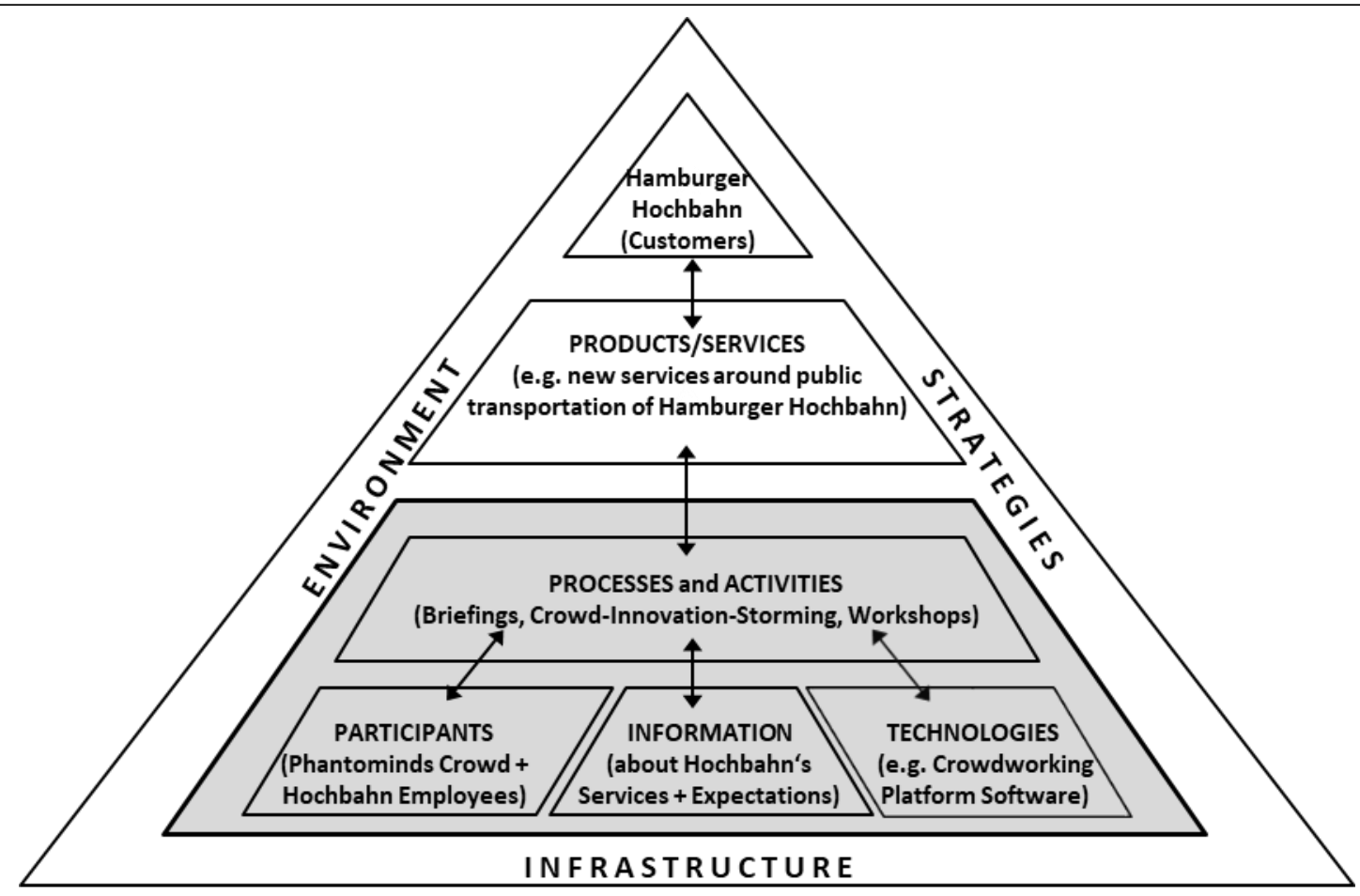

Figure 1. Depiction of the Hochbahn \& Phantominds Work System based on Alter ([2], p. 78) 
systems via crowdworking platform from this case ( $\mathrm{sec}$ tion 5.5.).

\subsection{Analysis of the work system}

As already stated above, a close analysis of the work system is key to better understand how its management took place in this case. And to derive adequate recommendations (for the general depiction and explanation of a work system according to Alter [2], see section 2). In this case, participants of the work system are on the one hand Phantominds' crowd workers, on the other hand also internal employees of Hamburger Hochbahn since they also worked on the concepts and solutions (for more details, see section 5.4). Information included e.g. the briefing for the crowd with details about Hamburger Hochbahn's current offerings for the customer and expectations for the results of this project. Technologies used by the work system are first of all the crowdworking platform Phantominds including its possibilities and supporting options for communication and collaboration. Processes and activities include (among others) the briefing, the crowdstorming and the workshops conducted. The whole work system of Hamburger Hochbahn and Phantominds (see the shaded area) plus its "surrounding" such as environment, infrastructure and strategies is visualized in figure 1 .

\subsection{Depiction of the general innovation process}

An accurate depiction of the process is key to better understand the success factors for the management of complex work systems via crowdworking platforms. In general, the first step in Phantominds' innovation process is the analysis of the challenge. Phantominds does this in personal conversations with the customer. This also includes an analysis for the customer company based on new technological trends, changing markets and new competitors. Together with the customer, Phantominds subsequently defines the project goals, the assignment for the crowd innovation project and the perspectives and target groups that need to be integrated. This results in a briefing of the project for Phantominds' crowd workers. The second step starts with the placement of the assignment on the crowdworking platform. During the period the project runs, the crowd community receives incentives both for delivering ideas and for providing improvement suggestions for other ideas. This allows to continuously develop and improve ideas in parallel. Providing incentives not only for own ideas, but also for improvement suggestions for ideas of other crowd workers, is furthermore a measure that also helps to foster collaboration among the crowd workers - according to our findings an important success factor especially when it comes to the management of complex work systems. Especially this exchange of ideas among crowd workers contributes heavily to the delivery of high-quality solutions for the customer since the communication among highly innovative crowd workers proofed to be very fruitful in the case of Hamburger Hochbahn and Phantominds. The contest duration was purposefully set by Phantominds using a period that allowed both for sufficient exchange between the solution providers (crowd workers) and at the same time for a timely delivery to the customer to allow a prompt implementation.

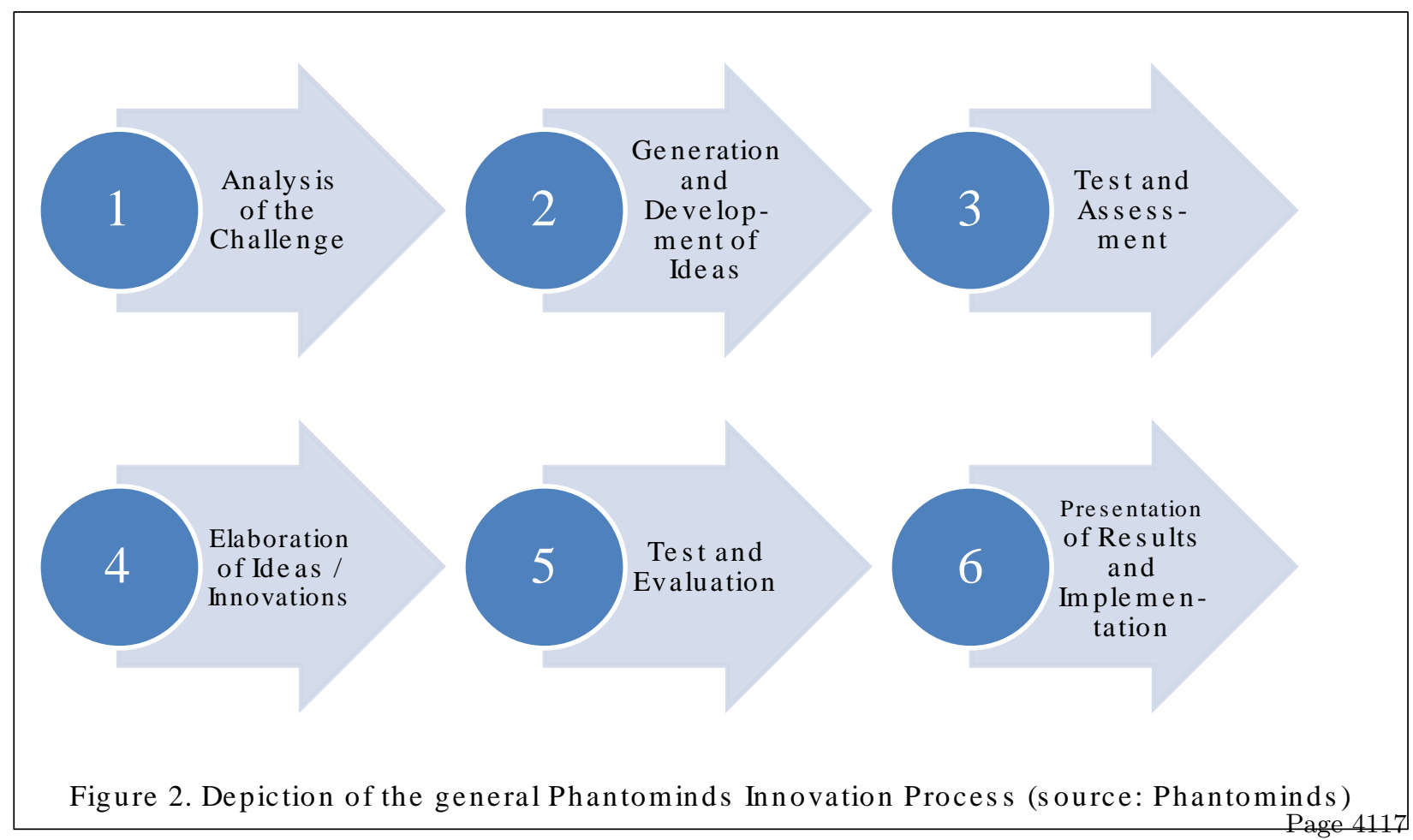


The third step includes the test and assessment of the developed ideas by the crowd community based on four quality criteria: (1) Novelty, (2) impact, (3) feasibility and (4) elaboration. This allows to choose the ideas with the highest potential regarding further development and feasibility. Alternatively, companies can also make their choice themselves, for example by using an external jury. The fourth step includes the concrete elaboration of the chosen ideas under consideration of the "fit-to-organization". Only crowd workers who have worked on the original idea are allowed to participate in this step. Phantominds also integrates internal employees of the customer company by request to ensure the feasibility of the suggestions. In the fifth step, the developed final solutions are again evaluated with a focus especially on quality. The customer decides if the crowd community or the company itself selects the best innovations/solutions. Finally, in the sixths and last step, the ideas/innovations/solutions are presented as a concept including defined execution steps to the customer. Phantominds also accompanies the execution itself by request. Figure 2 depicts these general six process steps.

\subsection{The Lean Integrated Innovation (LIC) Method}

Since existing methods did not fit the requirements of Phantominds with their business model based on a combination of virtual crowd-idea projects where the crowd workers collaboratively develop innovations ("co-creation") and the approach to deeply integrate the customer in the innovation project from the very beginning ("customer centricity"), the company developed an own method: The Lean Integrated Collaboration (LIC) Method. It comprises three approaches: The core of the method is based on the lean start-up movement, i.e. conduction of the development via hypothesis leaded and ongoing interactions with (potential) customers. This is also reflected in Phantominds's innovation process (see section 5.2): The idea- and co-creation phases are followed by test- and evaluation-phases to secure the quality of the developments and at the same time explore the market acceptance. The method allows to advance in several 'loops' to achieve a higher quality and feasibility of the developed innovations. The integration of external and internal perspectives during the innovation process is also an elementary part of the Phantominds crowdsourcing approach. External perspectives include the integration of future and already existing customers and their stakeholders such as employees and suppliers as well as external creatives and experts in the process. Last but not least, collaboration of the crowd workers during the innovation development process secures better quality and a higher degree of feasibility of the solutions and innovations.

\subsection{Additional aspects in the Hamburger Hoch- bahn project}

Since we already described the general process of Phantominds in the sections above, we will now focus regarding the project with Hamburger Hochbahn mainly on points that either differ from that approach or provide additional information. Hamburger Hochbahn got contact to Phantominds when key persons of both companies met at a conference event in Hamburg. The company first advertised the assignment, Phantominds won the order. At the meeting to discuss this joint project, Hochbahn and Phantominds furthermore agreed upon the point that the community itself should evaluate and select the best solutions (with Hamburger Hochbahn retaining the right to also further develop other solutions that have not been selected by the crowd). This and further details resulted in a detailed briefing for the crowd. Phantominds also customized the crowd-interface for Hamburger Hochbahn. Regarding the evaluation, all participating crowd workers (other crowd workers have not been allowed to) could give their ratings to the more than 130 solutions that have been delivered. This relates to the processing step of Crowd Capital [27] since Phantominds and their internal employees filter and integrate the incoming data, information and solutions to create value for the customer. To gain a large and qualified crowd, Phantominds announced this project also via their newsletter, their social media channels (e.g. Facebook and Twitter) and press releases with the possibility to register directly via their platform. The company provided financial rewards of 1,000 Euro each for the best three solutions from the crowd (in addition to the compensation that the platform provider Phantominds received itself). The project started in January 2016 and ran over several weeks. Phantominds moderated the process, commented on every idea and requested additional information from the customer Hamburger Hochbahn if required by the crowd. Altogether, the crowd of at that time more than 5,000 crowd workers provided 137 solutions. Duplicates or solutions that have been simply copied from other participants had been removed by Phantominds. In addition to moderating the whole process, Phantominds also asked questions if something had not been clear regarding first drafts from the crowd workers.

To give also non-monetary incentives, Phantominds used a level approach that allowed crowd workers to "climb the ranks" in four steps: "Rookie", "talent", "professional" and "all star". After the "crowdstorming" phase had ended, the best three solutions have been selected (within a week). This was done by assigning by the crowd workers one to five "stars" to every solution for each of the four criteria described in section 5.2. The overall rating has been automatically calculated of these four votings for these criteria (equally weighted). Afterwards, Phantominds compiled a holistic overview of all solutions delivered and provided a presentation for Page 4118 
Hamburger Hochbahn at a meeting that was also attended by two board members of the company. In the second quarter of 2016, Phantominds worked subsequently with internal employees of Hamburger Hochbahn to create first prototypes for four ideas (e.g. a mock-up app) and to test solutions with selected customers of the company. Even though a great part of the work in the solution creation phase had taken place electronically at the crowdworking platform, a great part of the later phases now took place in the "real world". One reason for this is according to our findings from the case that the more complex work is, the more likely it is also that "personal face-to-face interactions" are necessary (often in addition to interactions on the platform). Finally, Phantominds forwarded the further developed and refined solutions to its customer Hamburger Hochbahn.

\subsection{Derived recommendations for the manage- ment of complex work systems}

Using the "best practices" from the seminal project of Hamburger Hochbahn with Phantominds, we derive several key recommendations for the successful management of complex work systems via crowdworking platforms. To do so, we draw especially on measures communicated as key for the successful management of complex work systems during the interviews with the
Managing Director of Phantominds, Dr. Mirko Bendig. These measures take into account several characteristics of crowdworking platforms that add to the fact that managing work systems via such platforms differs from the management of complex work systems in "conventional" organizations: In contrast to internal employees of a company, crowd workers do not have to follow "instructions", but can choose on their own if they even want to participate in the respective project. Their education and skill level might be very heterogeneous compared to that of internal employees of a company which are more likely to be selected along the same criteria. Crowd workers are also not necessarily known by the customer company or even the crowdworking platform since many platforms allow using alias names. The exchange of information does often take place using technological infrastructure provided by the crowdworking platform. The derived measures also take into account the differences to the management of rather simple work systems via crowdworking platforms (e.g. less interdependencies, fewer stakeholders, less cognitive load, lower educations and skills necessary, etc./for more details, see section 2).

"The more complex the work system is, the more important is the question for the platform 'which is the right crowd for us that is able to proceed this work'? We employ great efforts to win the 'right' crowd for our

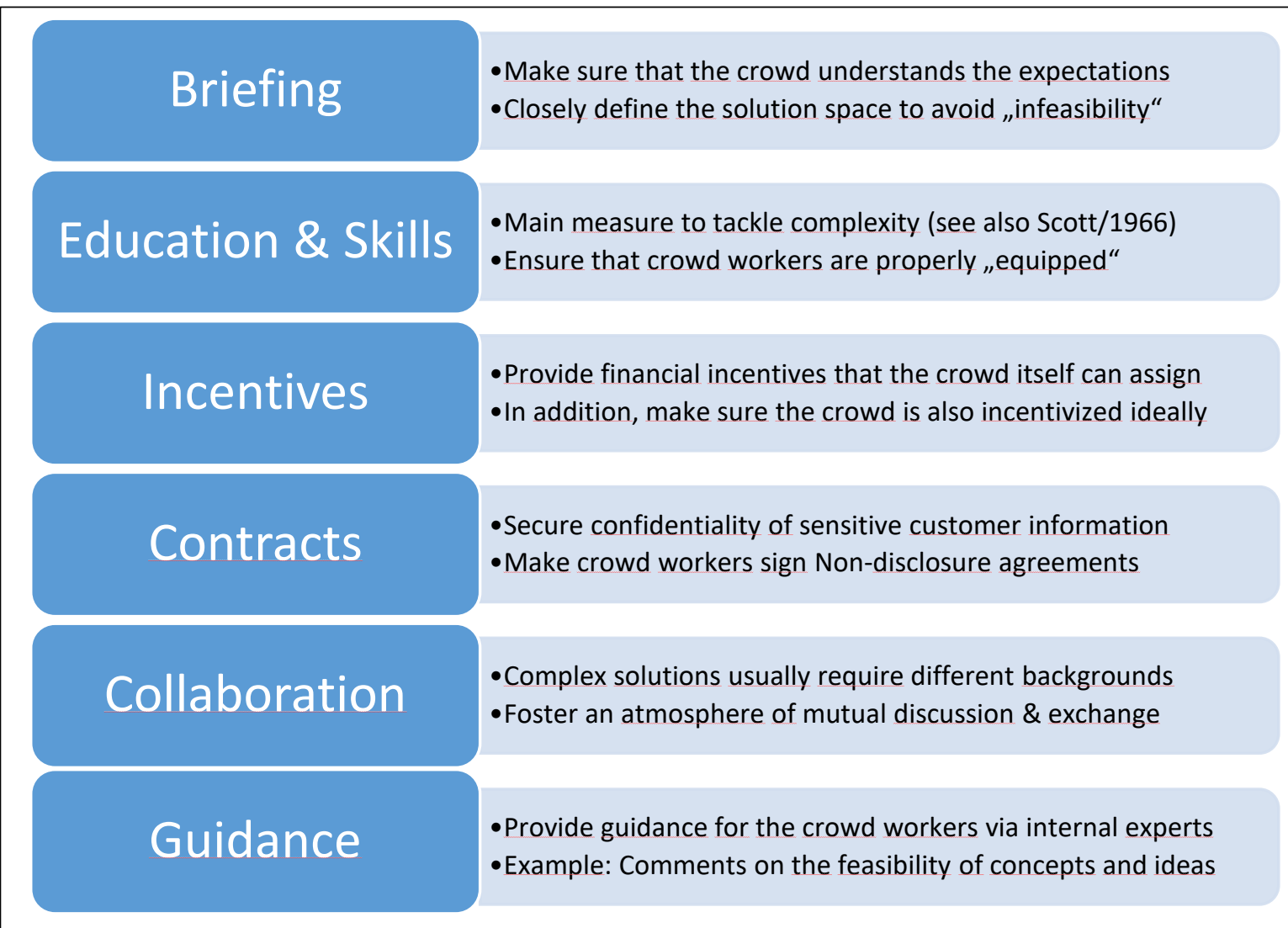

Figure 3. Six key recommendations for managing complex work systems (own depiction) 
platform. To do so, we for example use algorithms in open social media channels such as Twitter to determine which people have a high affinity for a certain theme and therefore could possibly provide valuable contributions or skills for our respective project."

Dr. Mirko Bendig,

Managing Director Phantominds

Figure 3 shows our derived six key measures as recommendations for the successful management of complex work systems via crowdworking platforms. First and foremost, a proper briefing for the crowd is key to ensure that crowd workers understand the expectations and the solutions space well. According to the interviews, this proved to be an important prerequisite for the success of the project with Hamburger Hochbahn. In consistence with organizational literature (see e.g. [29]), education and skills of the crowd workers and the necessity that these are "properly equipped" are also main success factors when it comes to the management of complex work systems. In addition to financial incentives, non-financial incentives such as being able to present ideas to high caliber personalities also had a huge impact on the motivation of the crowd. It adds to the "visibility" of crowd workers and gives them a sense of belongingness. Since complex work is more likely to be of strategic importance for organizations than for example simple microtask work, contracts that secure the confidentiality of information are key to gain the trust of a customers such as Hamburger Hochbahn and to subsequently be able to successfully conduct such a project. In contrast to rather simple work (for example picture tagging on microtaks platforms), complex work usually requires collaboration among participants with different backgrounds to add different views and work on different aspects of a solution. Especially regarding the innovation work in the case of Hamburger Hochbahn, collaboration among participants proved to enhance the quality of the solutions. According to the Managing Director of Phantominds, a key success factor has also been to guide the whole process from the beginning via internal experts of Phantominds. This allowed to comment on first concepts and ideas already in early work phases. Phantominds was therefore able to put an emphasis on feasibility and to keep possible future solutions "on track".

\section{Discussion and conclusion}

Crowdworking platforms have evolved as an innovative instrument and new form how to organize work. Nevertheless, many of these platforms currently manage rather simple work systems. Because of the three reasons we mentioned in the introduction of this paper, the business model of these platforms could come under pressure soon. Therefore, it is worth to investigate how complex work systems can be managed via such crowdworking platforms. The complexity of the work is a good proxy for the complexity of the work systems necessary to process that work since complex work is way more likely to for example include collaborations among participants. Collaboration among participants in turn adds to the complexitiy of the management of the work system compared to simple work where no collaboration among participants is required. Complex work also adds to the complexitiy of the "processes and activities"realm of the respective work system [2].

Using the case of the German Hamburger Hochbahn AG and the innovation platform Phantominds, we explored in this paper measures necessary for crowdworking platforms to be able to also manage such complex work systems. To derive proper recommendations, we analyzed the work system of Hamburger Hochbahn and Phantominds, explored the interplay between the crowd, the customer Hamburger Hochbahn and the platform provider Phantominds and subsequently delivered key recommendations for both companies that would like to use crowdworking platforms for the processing of work and platform operators. These recommendations address especially the areas "processes and activities" and "participants" of Alter's work system framework [2]. With this paper, we extend current knowledge in the realms of IS, organizational theory, and platform ecosystems by shedding light on a phenomenon that has not been in the main focus of research in these areas yet. We also contribute to practice since organizations such as crowdworking platforms gain insights on how to successfully manage complex work systems via crowdworking platforms and therefore are able to both extend and strengthen their business model.

\section{Future research}

As it is the case with every research, our work also faces limitations. Even though revelatory single-case studies can offer valuable contributions and insights, especially if an issue has not been investigated much (see also [34]), there is the need for further case study research about the management of complex work systems via crowdworking platforms. For example, case studies from the realms of engineering, IT or financial services could also provide valuable insights for this topic. Thy would allow to broaden the basis for recommendations. The key measures communicated as recommendations in section 5 have proven to be valid in this case. There might be further key measures that would prove to work in other industries and settings. We plan to adress these issues with our future research in this realm. Particularly, we will conduct further case studies about companies and projects that from our perspective also provide valuable information for the successful management of complex work systems via crowdworking platforms. Furthermore, we will conduct a workshop with crowdworking platform providers to gain additional insights and to evaluate our findings.

Page 4120 


\section{Acknowledgements}

This paper presents research that was conducted in the context of the project "Challenge cloud and crowd" that is funded by the German Federal Ministry of Education and Research (BMBF) within the program "Innovations for Tomorrow's Production, Services, and Work" (funding number: 02K14A071) and managed by the Project Management Agency Karlsruhe (PTKA).

\section{References}

[1] Afuah, A. and Tucci, C. L. 2012. Crowdsourcing as a Solution to Distant Search. Academy of Management Review 37, 3, 355-375.

[2] Alter, S. 2013. Work System Theory: Overview of Core Concepts, Extensions, and Challenges for the Future. Journal of the Association for Information Systems (JAIS) 14, 2, 72-121.

[3] Ashby, W. R. 1956. The Effect of Experience on a Determinate Dynamic System. Behavioral Science 1, 1, 35-42.

[4] Beer, S. 1967. Cybernetics and Management. John Wiley, New York.

[5] Benner, C., Ed. 2014. Crowdwork - zurück in die Zukunft? Perspektiven digitaler Arbeit. Bund-Verlag $\mathrm{GmbH}$, Frankfurt am Main.

[6] Boudreau, K., Gaule, P., Lakhani, K. R., Riedl, C., and Williams Woolley, A. 2014. From Crowds to Collaborators: Initiating Effort \& Catalyzing Interactions Among Online Creative Workers. Working Paper 14060. Harvard Business School.

[7] Boulding, K. E. 1956. General Systems Theory-The Skeleton of Science. Management Science 2, 3, 197208.

[8] Brynjolfsson, E. and McAfee, A. 2011. Race against the machine. How the digital revolution is accelerating innovation, driving productivity, and irreversibly transforming employment and the economy. Digital Frontier Press, Lexington, Mass.

[9] Däubler, W. and Klebe, T. 2015. Crowdwork: Die neue Form der Arbeit - Arbeitgeber auf der Flucht? Neue Zeitschrift für Arbeitsrecht (NZA), 17, 1032-1041.

[10] Eisenhardt, K. M. 1989. Building Theories from Case Study Research. The Academy of Management Review $14,4,532-550$.

[11] Fayol, H. 1949. General and Industrial Management. Pitman, London.

[12] Ford, M. 2015. Rise of the Robots. Technology and the Threat of a Jobless Future. Basic Books, New York.

[13] Frey, C. B. and Osborne, M. A. 2013. The Future of Employment: How Susceptible are Jobs to Computerisation? Oxford Martin School Publications.

[14] Howe, J. 2006. The Rise of Crowdsourcing. Wired Magazine 14, 6, 176-183.

[15] Kittur, A., Smus, B., Khamkar, S., and Kraut, R. E. 2011. CrowdForge: Crowdsourcing Complex Work. Proceedings of the 24th annual ACM Symposium on User Interface Software and Technology: October 1619, 2011, Santa Barbara, CA/USA.

[16] Kuek, S. C., Paradi-Guilford, C. M., Fayomi, T., Imaizumi, S., and Ipeirotis, P. 2015. The Global Opportunity in Online Outsourcing. The World Bank, Washington.
[17] Leimeister, J. M. and Zogaj, S. 2013. Neue Arbeitsorganisation durch Crowdsourcing. Eine Literaturstudie. Arbeitspapier 287, Düsseldorf.

[18] Mrass, V., Li, M. M., and Peters, C. 2017. Towards a Taxonomy of Digital Work. 25. European Conference on Information Systems (ECIS), Guimarães, Portugal, 2017, 2515-2524.

[19] Mrass, V. and Peters, C. 2017. Crowdworking-Plattformen in Deutschland. Working Paper Series 16. University of Kassel, Kassel.

[20] Mrass, V., Peters, C., and Leimeister, J. M. 2016. New Work Organization through Crowdworking Platforms. A Case Study. Konferenz "Zukunftsprojekt Arbeitswelt 4.0", 19.09.2016, Stuttgart.

[21] Mrass, V., Peters, C., and Leimeister, J. M. 2017. One for All? Managing External and Internal Crowds through a Single Platform - A Case Study. 50th Hawaii International Conference on System Sciences (HICSS), 2017, 4324-4333.

[22] Mrass, V., Peters, C., and Leimeister, J. M. 2017. Von Kunden für Kunden: Crowd Services als Erweiterung der Digital Customer Experience. HMD Praxis der Wirtschaftsinformatik 54, 317.

[23] Perrow, C. 1982. Three Mile Island: A Normal Accident. The international yearbook of organization studies, $1-25$.

[24] Prpic, J. 2017. Specifying and Operationalizing an Organizational Theory of Crowdsourcing. Doctoral Thesis, Luleå University of Technology.

[25] Prpic, J., Taeihagh, A., and Melton, J. 2015. The Fundamentals of Policy Crowdsourcing. Policy \& Internet 7, 3, 340-361.

[26] Prpić, J. and Shukla, P. Crowd Science: Measurements, Models, and Methods. In 2016 49th Hawaii International Conference on System Sciences (HICSS), 43654374.

[27] Prpić, J. and Shukla, P. The Theory of Crowd Capital. In 2013 46th Hawaii International Conference on System Sciences (HICSS), 3505-3514.

[28] Prpić, J., Shukla, P. P., Kietzmann, J. H., and McCarthy, I. P. 2015. How to work a crowd. Developing crowd capital through crowdsourcing. Business Horizons 58, 1, 77-85.

[29] Scott, W. R. 1966. Professionals in Bureaucracies - Areas of Conflict. Professionalization, 265-275.

[30] Scott, W. R. and Davis, G. F. 2016. Organizations and organizing. Rational, natural, and open systems perspectives. Routledge, London, New York.

[31] Stinchcombe, A. L. 1990. Information and Organizations. University of California Press, Berkeley.

[32] Surowiecki, J. 2004. The wisdom of crowds. Why the many are smarter than the few and how collective wisdom shapes business, economies, societies, and nations. Doubleday, New York.

[33] Tiwana, A., Konsynski, B., and Venkatraman, N. 2013. Special Issue. Information Technology and Organizational Governance: The IT Governance Cube. Journal of Management Information Systems (JMIS) 30, 3, $7-$ 12.

[34] Yin, R. K. 2014. Case Study Research. Design and Methods. SAGE Publications, Inc., Los Angeles. 